\title{
Absorption and scattering by long and randomly oriented linear chains of spheres
}

\author{
Euntaek Lee and Laurent Pilon* \\ Mechanical and Aerospace Engineering Department, Henry Samueli School of Engineering and Applied Science, \\ University of California, Los Angeles, 420 Westwood Plaza, Eng. IV 37-132, Los Angeles, California 90095, USA \\ ${ }^{*}$ Corresponding author: pilon@seas.ucla.edu
}

Received June 13, 2013; revised August 2, 2013; accepted August 2, 2013; posted August 5, 2013 (Doc. ID 191942); published August 30, 2013

\begin{abstract}
This paper demonstrates that the scattering cross section per unit length of randomly oriented linear chains of optically soft spheres asymptotically converges toward those of randomly oriented and infinitely long cylinders with volume-equivalent diameter as the number of spheres increases. The critical number of spheres necessary to approximate the linear chains of spheres as infinitely long cylinders decreased rapidly as the size parameter of an individual sphere increased from 0.01 to 10. On the other hand, their absorption cross section per unit length was identical to that of an infinitely long volume-equivalent cylinder for any number of spheres. However, this approximation does not apply to the angle-dependent normalized Stokes scattering matrix element ratios. (c) 2013 Optical Society of America

OCIS codes: $\quad$ (290.5825) Scattering theory; (290.5855) Scattering, polarization; (290.7050) Turbid media;

(100.3200) Inverse scattering.

http://dx.doi.org/10.1364/JOSAA.30.001892
\end{abstract}

\section{INTRODUCTION}

Light absorption and scattering by nonspherical particles or by clusters of spheres have been the subject of great interest in the radiation transfer community and have found various applications in science and engineering ranging from astrophysics and atmospheric science to combustion systems and aerosol-based processes [1-4] . Numerical tools have been developed to predict light absorption and scattering by nonspherical scatterers based on (i) the T-matrix methods [ $\underline{5}-10]$, (ii) the discrete-dipole approximation (DDA) [11-13], or (iii) the finite-difference time-domain method $[\underline{3,14}, \underline{15}]$, to name the most widely used. Similarly, light absorption and scattering by a cluster or aggregate of spheres have been predicted by (i) the superposition T-matrix method [16-23], (ii) the DDA [24], and (iii) the volume-integral equation combined with the method of moments [25-27]. Most of the studies on sphere clusters focused on radiation scattering and absorption by soot particles.

Depending on the size and morphology of the scatterers and on the wavelength, calculations can be time consuming and require large computing resources, regardless of the method used. Moreover, actual particles may not have welldefined shapes. Thus, for practical purposes, it is important to try to find simplified models to approximate scatterers with complex geometries as equivalent particles with simpler shapes such as spheres or cylinders [15,28]. For example, Kahnert et al. [28] showed that the extinction and scattering cross sections, the single scattering albedo, and the asymmetry factor of an ensemble of randomly oriented polyhedral prisms with power-law size distribution and size comparable to the wavelength of light can be approximated as an ensemble of spheres, spheroids, or finite-length cylinders with the same volume, complex index of refraction, and size distribution. Note that treating the prisms as volume-equivalent cylinders or spheroids gave slightly better results than treating them as spheres. However, any of these simplifications failed to predict the linear depolarization ratio. Similarly, Yang et al. [15] investigated the single scattering properties of various Platonic particles and compared their radiation characteristics with those of equivalent spheres having the same (i) geometric dimension, (ii) surface area, (iii) volume, or (iv) volume-to-surface area ratio. The authors concluded that all these approximations led to significant errors in the extinction efficiency factor, single scattering, albedo, and/or scattering matrix elements. The volume-equivalent spheres gave the smallest errors of all equivalent spheres considered. In addition, approximating the Platonic particles by their volume-tosurface area ratio equivalent sphere led to the largest errors.

The goal of the present study is to theoretically identify simplified models for predicting light absorption and scattering by long and randomly oriented linear chains of spheres. This question finds its motivation in predicting light transfer in photobioreactors cultivating photosynthetic filamentous cyanobacteria for wastewater treatment, sustainable biofuel, and/ or fertilizer productions [29]. It also applies to the field of ocean optics for remote sensing applications and for studying carbon dioxide and nitrogen cycles [4], for example.

\section{BACKGROUND}

\section{A. Filamentous Cyanobacteria}

Cyanobacteria, also known as blue-green algae, are photoautotrophic prokaryotes that are capable of conducting oxygenic photosynthesis [30]. They use solar radiation in the photosynthetically active radiation (PAR) region, defined by wavelength ranging from 400 to $700 \mathrm{~nm}$, as their energy source. They can be found in nearly every terrestrial and 
aquatic habitat on Earth and are responsible for the presence of oxygen in the atmosphere [31]. Cyanobacteria can be unicellular and filamentous, and their size can range from 0.5 to $40 \mu \mathrm{m}$ in diameter depending on the strain [30]. Some filamentous forms have evolved to contain the nitrogenase enzyme in specialized nitrogen-fixing cells called heterocysts. Several species can also produce hydrogen $\mathrm{H}_{2}$ through direct and indirect biophotolysis and have been considered for photobiological hydrogen production [32].

Figure 1 shows micrographs of different species of filamentous cyanobacteria and illustrates, in particular, (a) nearly spherical vegetative cells of the nitrogen-fixing cyanobacteria Nostoc punctiforme 5-6 $\mu \mathrm{m}$ in diameter, (b) an ensemble of Nostoc punctiforme showing filaments with both vegetative (5-6 $\mu \mathrm{m}$ in diameter) and heterocyst cells $(6-10 \mu \mathrm{m}$ in diameter), (c) an individual filament of Anaebena sp. with spherical to oblong vegetative cells 4-14 $\mu \mathrm{m}$ in diameter, and (d) aggregating filaments of Anabaena iyengari. Other filamentous cyanobacteria with similar morphology include Anabaena sphaerica, Anabaena cylindrica, Anabaena variabilis, and Anabaena azollae, to name a few.

\section{B. Scattering Matrix}

The radiation incident on a particle of arbitrary shape at location $\mathbf{r}$ can be represented by the incident Stokes vector $\mathbf{I}_{\text {inc }}\left(\mathbf{r}, \hat{s}_{i}\right)=\left(I_{\text {inc }}, Q_{\text {inc }}, U_{\text {inc }}, V_{\text {inc }}\right)^{T}$, where $I, Q, U$, and $V$ are the so-called Stokes parameters [33]. The Stokes vector of the scattered radiation denoted by $\mathbf{I}_{\text {sca }}(\mathbf{r}, \hat{s})=$ $\left(I_{\text {sca }}, Q_{\text {sca }}, U_{\text {sca }}, V_{\text {sca }}\right)^{T}$ is related to the incident Stokes vector by the Mueller matrix $[Z(\Theta)]$ according to $[\underline{18}]$

$$
\mathbf{I}_{\mathrm{sca}}(\mathbf{r}, \hat{s})=\frac{1}{r^{2}}[\mathbf{Z}(\Theta)] \mathbf{I}_{\mathrm{inc}}\left(\mathbf{r}, \hat{s}_{i}\right),
$$

where $r$ is the norm of the location vector $\mathbf{r}$, and $\Theta$ is the socalled scattering angle ranging from $0^{\circ}$ to $180^{\circ}$ and defined as the angle between the incident and scattered directions
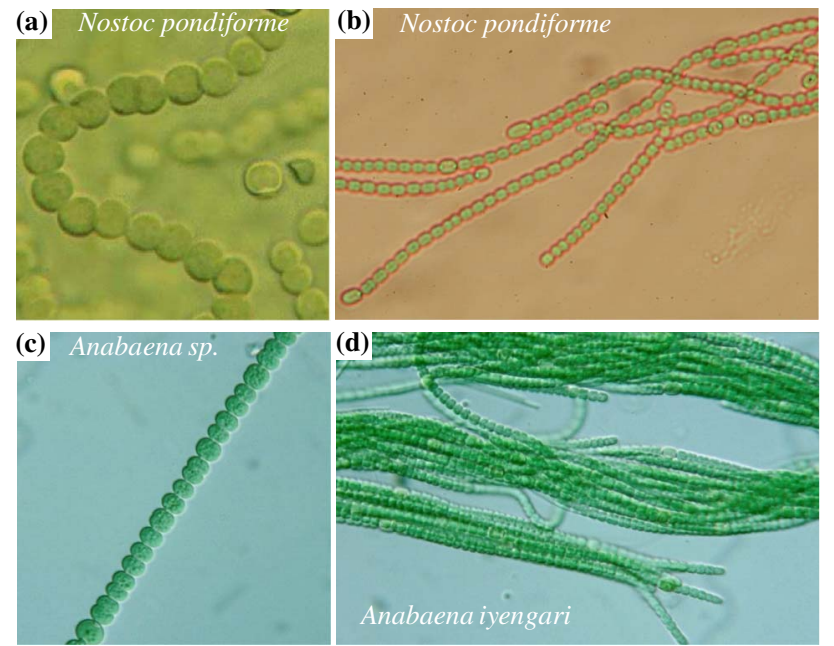

Fig. 1. Micrographs of filamentous cyanobacteria: (a), (b) Nostoc pondiforme, (c) Anabaena sp., and (d) Anabaena iyengari. Reproduced with permission from (a) Isao Inouye (University of Tsukuba), Mark Schneegurt (Wichita State University), and Cyanosite (www-cyanosite.bio.purdue.edu); (b) Prof. Ann Magnuson (Uppsala University); and (c), (d) Prof. Yuuji Tsukii (Hosei University, http:// protist.i.hosei.ac.jp/). denoted by $\hat{s}_{i}$ and $\hat{s}$, respectively. For a cluster of particles with a plane of symmetry, it is convenient to use the normalized (or Stokes) scattering matrix expressed as [18]

$$
[\mathbf{F}(\Theta)]=\frac{4 \pi}{C_{\text {sca }}}[\mathbf{Z}(\Theta)]
$$

where $C_{\text {sca }}$ is the particle's scattering cross section. Then, the normalized Stokes scattering matrix has a $4 \times 4$ structure with six independent elements and can be written as [2]

$$
[\mathbf{F}(\Theta)]=\left[\begin{array}{cccc}
F_{11}(\Theta) & F_{12}(\Theta) & 0 & 0 \\
F_{12}(\Theta) & F_{22}(\Theta) & 0 & 0 \\
0 & 0 & F_{33}(\Theta) & F_{34}(\Theta) \\
0 & 0 & -F_{34}(\Theta) & F_{44}(\Theta)
\end{array}\right]
$$

The normalized Stokes scattering matrix element $F_{11}(\Theta)$ is the scattering phase function normalized according to

$$
\frac{1}{4 \pi} \int_{4 \pi} F_{11}(\Theta) \mathrm{d} \Omega=1
$$

where $\Omega$ is the solid angle around the scattering angle $\Theta$. In addition, the first moment of the scattering phase function is the so-called asymmetry factor defined as [29]

$$
g=\frac{1}{4 \pi} \int_{4 \pi} F_{11}(\Theta) \cos \Theta \mathrm{d} \Omega
$$

It describes the shape of the scattering phase function and is equal to 0.0 for isotropic scattering and -1 and 1 for purely backward and forward scattering, respectively.

The ratio $-F_{12}(\Theta) / F_{11}(\Theta)$ represents the degree of linear polarization of the scattered radiation for unpolarized incident radiation [34]. The ratio $F_{22}(\Theta) / F_{11}(\Theta)$ captures the nonsphericity of the particles and is equal to unity for a single sphere [34]. Other indicators of the sphericity of the scatterer are the linear and circular polarization ratios, respectively, defined as [18]

$$
\delta_{L}=\frac{\left[F_{11}\left(180^{\circ}\right)-F_{22}\left(180^{\circ}\right)\right]}{\left[F_{11}\left(180^{\circ}\right)+F_{22}\left(180^{\circ}\right)\right]}
$$

and

$$
\delta_{C}=\frac{\left[F_{11}\left(180^{\circ}\right)+F_{44}\left(180^{\circ}\right)\right]}{\left[F_{11}\left(180^{\circ}\right)-F_{44}\left(180^{\circ}\right)\right]} .
$$

For a single sphere, $\delta_{C, s}=\delta_{L, s}=0$ [18], while for randomly oriented rotationally symmetric particles, $\delta_{C} \geq 2 \delta_{L}$ [6]. For a randomly oriented and infinitely long cylinder, they both vanish, i.e., $\delta_{C, c}=\delta_{L, c}=0$. The term $F_{34}$ represents how much incident radiation obliquely polarized at $45^{\circ}$ gets transformed into circularly polarized radiation [34]. Hovenier and Mackowski [35] derived relations between scattering matrix elements at forward and backward scattering directions $(\Theta=0$ and $180^{\circ}$ ) for randomly oriented single particle and a cluster of particles with one plane of symmetry and for rotationally symmetric particles. They showed that, for such particles or clusters, $\Delta\left(0^{\circ}\right)=F_{11}\left(0^{\circ}\right)-F_{22}\left(0^{\circ}\right)-F_{33}\left(0^{\circ}\right)+F_{44}\left(0^{\circ}\right)$ was equal to zero and that $F_{11}\left(180^{\circ}\right)-2 F_{22}\left(180^{\circ}\right)=F_{44}\left(180^{\circ}\right)$. 
These relationships were validated using the T-matrix method. Finally, for a spherical scatterer $F_{22}(\Theta)=F_{11}(\Theta)$ and $F_{33}(\Theta)=F_{44}(\Theta)$ [33].

\section{T-matrix Method for Linear Chain of Spheres}

The superposition T-matrix method has been developed for arbitrary clusters of multiple spheres as described in detail in [17]. This approach is based on the superposition principles whereby the scattered field from the entire cluster of spheres is estimated by summing those from each of the spheres [17]. The scattered fields in sphere-centered coordinates are also transformed into cluster-centered coordinates [17]. The absorption and scattering cross sections and efficiency factors for randomly oriented clusters of identical spheres can be obtained by using the matrix relationships for the scattered and incident field and integrating the incident field over all propagation directions and polarizations [22]. The corresponding normalized Stokes scattering matrix can be obtained analytically from operations on the T-matrix [22].

Figure 2(a) illustrates absorption and scattering by a linear chain of monodisperse spheres of complex index of refraction $m_{2}=n_{2}+i k_{2}$ in a nonabsorbing medium of refraction index $n_{1}$. Mackowski and Mishchenko [22] defined the orientationaveraged absorption and scattering cross sections of a cluster of $N_{s}$ monodisperse spheres, denoted by $\left\langle C_{\mathrm{abs}, s}\right\rangle\left(m, \chi_{s}, N_{s}\right)$ and $\left\langle C_{\text {sca }, s}\right\rangle\left(m, \chi_{s}, N_{s}\right)$, respectively, and expressed in $\mathrm{m}^{2}$ as

$$
\left\langle C_{\mathrm{abs} / \mathrm{sca}, s}\right\rangle=\frac{\pi d_{s, \mathrm{eq}, V}^{2}}{4}\left\langle Q_{\mathrm{abs} / \mathrm{sca}, s}\right\rangle,
$$

where $d_{s, \mathrm{eq}, V}$ is the equivalent diameter of a single sphere having volume identical to that of the cluster of $N_{s}$ monodisperse spheres of diameter $d_{s}$, i.e., $d_{s, \text { eq }, V}=d_{s} N_{s}^{1 / 3}$. Here, $m=m_{2} / n_{1}$ is the relative complex index of refraction of the spheres with respect to that of the nonabsorbing surrounding medium, while $\chi_{s}=\pi d_{s} / \lambda$ is the size parameter of a single sphere of diameter $d_{s}$. The notation $\langle X\rangle$ refers to the orientationally averaged property. The absorption and scattering efficiency factors denoted by $\left\langle Q_{\mathrm{abs}, s}\right\rangle\left(m, \chi_{s}, N_{s}\right)$ and $\left\langle Q_{\mathrm{sca}, s}\right\rangle\left(m, \chi_{s}, N_{s}\right)$ were computed by the T-matrix method.

Numerous studies have been concerned with light absorption and scattering by fractal aggregates of small spherical (a)

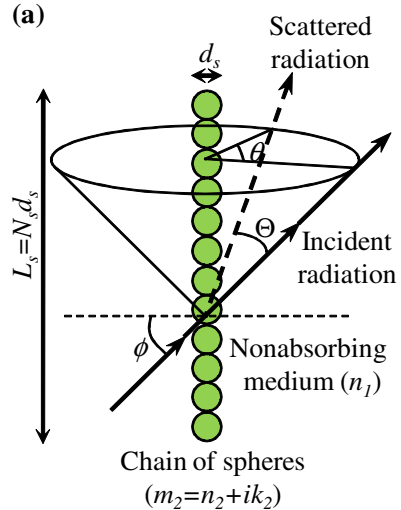

(b)

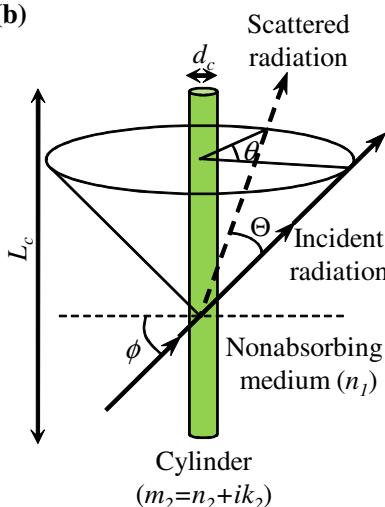

Fig. 2. Schematic and coordinate system associated with absorption and scattering of incident radiation at incident angle of $\phi$ by (a) linear chain of $N_{s}$ spheres of diameter $d_{s}$ with complex index of refraction $m_{2}=n_{2}+i k_{2}$ in a nonabsorbing medium of $m_{1}=n_{1}$, and (b) infinitely long cylinder of diameter $d_{c}$ [37]. particles simulating soot particles forming in combustion systems [21,25-27,36]. Mishchenko and Mackowski [16] demonstrated the use of the T-matrix method to determine the elements of the normalized Stokes scattering matrix for randomly oriented and connected bispheres. The authors extended this formulation to determine the elements of the scattering matrix of randomly oriented arbitrary clusters of spheres [20]. In particular, they considered linear chains of spheres consisting of 1 to 5 spheres with size parameter $\chi_{s}=$ 5 and relative complex index of refraction $m=1.5+i 0.005$ [20]. They concluded that increasing the number of spheres (i) enhanced scattering in the forward direction $\left(\Theta=0^{\circ}\right)$ and (ii) damped out the oscillations in the normalized Stokes scattering matrix elements as a function of scattering angle $\Theta$. In addition, the elements $F_{11}, F_{12}, F_{22}, F_{33}, F_{34}$, and $F_{44}$ became nearly independent of the number of spheres for chains consisting of two spheres or more. To illustrate their symmetry relations for forward and backward scattering by randomly oriented clusters of spheres with a plane of symmetry, Hovenier and Mackowski [35] considered a linear chain of spheres consisting of four spheres with size parameter $\chi_{s}=3$ and relative complex index of refraction $m=1.311+$ $i 3.11 \times 10^{-9}$ and showed that $\Delta\left(0^{\circ}\right)=0$ [20] .

\section{Absorption and Scattering by Infinitely Long Cylinders}

Figure 2(b) illustrates absorption and scattering by an infinitely long cylinder of diameter $d_{c}$ with complex index of refraction $m_{2}=n_{2}+i k_{2}$ in a nonabsorbing medium of refraction index $n_{1}$. Collimated radiation is incident onto the cylinder at an angle $\phi$ with respect to the normal of the cylinder axis [37]. The scattered radiation propagates along the conical surface defined by the apex angle of $\pi / 2-\phi$. The direction of the scattered radiation is defined azimuthally relative to the incident radiation by the angle $\theta$. The theory predicting the absorption and scattering cross sections of infinitely long cylinders is well established [1,2,37-41]. First, cylinders can be treated as infinitely long provided that their length $L_{c}$ is much larger than their diameter $d_{c}$, i.e., $L_{c} \gg d_{c}$ [2]. The extinction and scattering cross sections per unit length of an infinitely long cylinder of diameter $d_{c}$ with relative complex index of refraction $m=m_{2} / n_{1}$ for a given incident direction $\phi$ are denoted by $C_{\text {ext, } c}^{\prime}$ and $C_{\mathrm{sca}, c}^{\prime}$ and expressed in $\mathrm{m}^{2} / \mathrm{m}$. They are defined as [42]

$$
C_{\mathrm{ext} / \mathrm{sca}, c}^{\prime}\left(m, \chi_{c}, \phi\right)=2 d_{c} Q_{\mathrm{ext} / \mathrm{sca}, c}\left(m, \chi_{c}, \phi\right),
$$

where $\chi_{c}=\pi d_{c} / \lambda$ is the cylinder size parameter, while $Q_{\mathrm{ext}, c}\left(m, \chi_{c}, \phi\right)$ and $Q_{\mathrm{sca}, c}\left(m, \chi_{c}, \phi\right)$ are the extinction and scattering efficiency factors, respectively. The extinction and scattering cross sections $C_{\mathrm{ext}, c}^{\prime}\left(m, \chi_{c}, \phi\right)$ and $C_{\mathrm{sca}, c}^{\prime}\left(m, \chi_{c}, \phi\right)$ can be expressed in terms of the coefficients $a_{n}$ and $b_{n}$ given in terms of Bessel and Hankel functions [1,42]. In addition, the absorption cross sections per unit length are defined as $\left\langle C_{\mathrm{abs}, c}^{\prime}\right\rangle\left(m, \chi_{c}\right)=\left\langle C_{\mathrm{ext}, c}^{\prime}\right\rangle\left(m, \chi_{c}\right)-\left\langle C_{\mathrm{sca}, c}^{\prime}\right\rangle\left(m, \chi_{c}\right)$. The absorption and scattering cross sections per unit length of an infinitely long and randomly oriented cylinder are estimated by averaging the angular cross sections over the observation hemisphere according to [37] 


$$
\left\langle C_{\mathrm{abs} / \mathrm{sca}, c}^{\prime}\right\rangle\left(m, \chi_{c}\right)=\int_{0}^{\pi / 2} C_{\mathrm{abs} / \mathrm{sca}, c}^{\prime}\left(m, \chi_{c}, \phi\right) \cos \phi \mathrm{d} \phi .
$$

To the best of our knowledge, only a few studies have presented the normalized Stokes scattering matrix elements of linear chains of spheres $[20,35, \underline{43}]$. In addition, the number of spheres considered did not exceed 5 , and a single relatively large size parameter was investigated. By contrast, the present study investigates the effect of the sphere size parameter (ranging from 0.01 to 10) and the number of spheres (between 1 and 4000) on the absorption and scattering cross sections per unit length, the scattering phase function, and the normalized Stokes scattering matrix elements of linear chains of spheres. It aims to answer the following questions: (1) can one approximate long and randomly oriented linear chains of spheres as randomly oriented infinitely long cylinders? (2) If so, how long should the chains be or how many spheres should they consist of? And (3) what should the diameter of the equivalent cylinder be? Note that special emphasis was placed on optically soft particles characterized by a small mismatch between their complex index of refraction and the surrounding medium, i.e., $|m-1| \ll 1$.

\section{ANALYSIS}

\section{A. Problem Statement}

Radiation characteristics of photosynthetic microorganisms depend largely on their size, shape, pigment composition, internal structure, and effective optical properties [44]. They are essential in predicting light transfer in photobioreactors and the overall performance of the systems [29,45,46]. Microalgae and cyanobacteria feature small index mismatch with their surrounding medium and can be considered as optically soft [4]. In addition, the circularity and aspect ratio of individual cells in cyanobacteria filaments are not exactly unity as suggested by the micrographs shown in Fig. 1. However, the average aspect ratio of vegetative or heterocyst cells is typically less than 1.33. Our previous study showed that the radiation characteristics of randomly oriented spheroidal microalgae with aspect ratio less than 1.33 computed with the T-matrix method were nearly identical to those of surface-equivalent spheres with identical complex index of refraction computed by Lorenz-Mie theory [47]. These observations suggest that, as a first-order approximation, filamentous cyanobacteria can be approximated as linear chains of connected, spherical, and homogeneous cells. In a well-mixed suspension, they could further be treated as randomly oriented.

Transport of unpolarized light through well-mixed suspensions of a linear chain of spheres of known concentration is governed by the radiation transfer equation, requiring knowledge of their absorption and scattering cross sections $\left\langle C_{\text {abs, }, \lambda}\right\rangle$ and $\left\langle C_{\text {sca. } \lambda}\right\rangle$, and of the scattering phase function $F_{11}(\Theta)$. Moreover, investigating other elements of the scattering matrix could prove useful for remote sensing of these suspensions. Then, their radiation characteristics could be numerically predicted by the superposition T-matrix method [22]. However, these calculations can be very time consuming, particularly given the length and size of these microorganisms and the wavelength of light in the PAR region. Therefore, from a radiation standpoint, one may wonder if these microorganisms could be modeled as randomly oriented and infinitely long cylinders $[2, \underline{37}, \underline{40]}$.

\section{B. Methodology}

The computer code for the superposition T-matrix method used to predict absorption and scattering cross sections and normalized Stokes scattering matrix elements of randomly oriented linear chains of monodisperse spheres was obtained from [22]. It was successfully validated by comparing predictions of the absorption and scattering efficiency factors and the normalized Stokes scattering matrix elements predicted by our code with those (i) for randomly oriented bispheres with $m=1.5+i 0.005$ and $\chi_{s}=10$ reported by Mishchenko and Mackowski [19] and (ii) for randomly oriented linear chains of spheres composed of 1 to 5 touching spheres with $m=1.5+i 0.005$ and $\chi_{s}=5$ reported by Mackowski and Mishchenko [20]. Similarly, the code for predicting the absorption and scattering cross sections and the normalized Stokes scattering matrix of randomly oriented and infinitely long cylinders used in this study was obtained from [41]. It was successfully validated against the results reported by Lee $[37, \underline{48}]$ for the extinction efficiency factor and the scattering phase function of randomly oriented and infinitely long cylinders in vacuum.

Yang et al. [15] warned that it could be "misleading" to compare the efficiency factors of particles with complex shape with those of their equivalent spheres rather than directly comparing their cross sections. Indeed, radiation transfer calculations use cross sections and the particle number density $N_{T}$ (in $\# / \mathrm{m}^{3}$ ) to estimate the absorption and scattering coefficients as $\kappa_{\lambda}=C_{\mathrm{abs}, \lambda} N_{T}$ and $\sigma_{s, \lambda}=C_{\mathrm{sca}, \lambda} N_{T}$. These coefficients are used to compute the radiation intensity solution of the radiative transfer equation [42]. Thus, the present study compares the absorption and scattering cross sections of a randomly oriented linear chain of spheres and infinitely long cylinders. In order to directly compare the radiation cross sections of a randomly oriented linear chain composed of $N_{s}$ spheres of diameter $d_{s}$ with those of an infinitely long cylinder, their orientationally averaged scattering and absorption cross sections were defined per unit length of linear chain of spheres as

$$
\left\langle C_{\mathrm{abs} / \mathrm{sca}, s}^{\prime}\right\rangle=\frac{\left\langle C_{\mathrm{abs} / \mathrm{sca}, s}\right\rangle}{N_{s} d_{s}} .
$$

Finally, in the present study, the complex index of refraction of the spheres was taken as $m_{2}=1.355+i 0.004$, while that of the nonabsorbing surrounding medium was $n_{1}=1.333$. These optical properties were representative of cyanobacteria in suspension in their nutrient medium and exposed to visible light [ㄱ].

\section{RESULTS AND DISCUSSION}

\section{A. Absorption and Scattering Cross Sections}

Figure 3 shows the absorption cross section per unit length of a randomly oriented linear chain of monodisperse spheres as a function of the number of spheres $N_{s}$ for size parameter $\chi_{s}=0.01,0.1,1$, and 10 . The results were compared with those for an infinitely long cylinder with identical relative complex index of refraction. Two equivalent diameters were considered, assuming (1) the cylinder had the same surface area as the chain of spheres resulting in the surface-equivalent diameter $d_{c, \text { eq }, S}=d_{s}$ or (2) the cylinder had the same volume as the chain of spheres resulting in volume-equivalent 

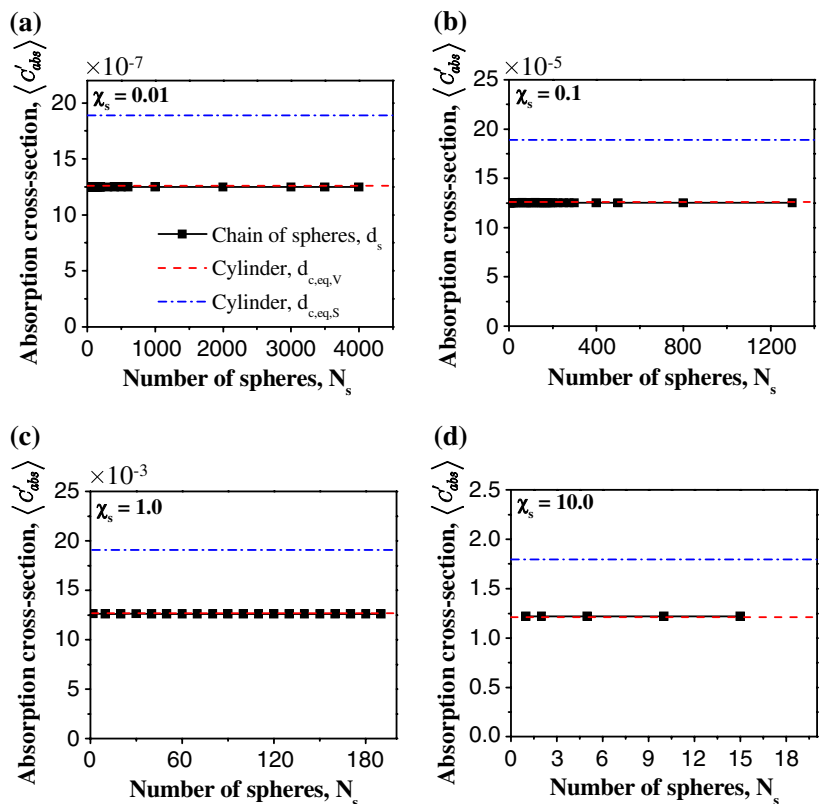

Fig. 3. Absorption cross section $\left\langle C^{\prime}{ }_{\mathrm{abs}}\right\rangle\left(m, \chi_{\mathrm{s}}\right)$ per unit length (in $\mathrm{m}^{2} / \mathrm{m}$ ) for randomly oriented linear chains of monodisperse spheres as a function of the number of spheres $N_{s}$ and for randomly oriented and infinitely long equivalent cylinders of diameter $d_{c, \mathrm{eq}, S}$ and $d_{c, \text { eq } . V}$ for size parameter $\chi_{s}=0.01,0.1,1.0$, and 10.0 and $m=1.0165+i 0.003$.

diameter $d_{c, \mathrm{eq}, V}=\sqrt{2 / 3} d_{s}$. Figure 3 indicates that the absorption cross sections per unit length of a randomly oriented linear chain of spheres were equal to those of randomly oriented and infinitely long cylinders with volume-equivalent diameter regardless of the number of spheres, i.e., $\left\langle C_{\mathrm{abs}, s}^{\prime}\right\rangle\left(m, \chi_{s}, N_{s}\right)=$ $\left\langle C_{\mathrm{abs}, c}^{\prime}\right\rangle\left(m, \chi_{c, \mathrm{eq}, V}\right)$. However, they were much smaller than those of a randomly oriented and infinitely long cylinder with surface-equivalent diameter $d_{c, \text { eq }, S}$.

Similarly, Fig. 4 shows the scattering cross section per unit length of a randomly oriented linear chain of spheres as a function of the number of spheres $N_{s}$. Four size parameters were considered, namely $\chi_{s}=0.01,0.1,1$, and 10 . These results were compared with the scattering cross section per unit length of a randomly oriented and infinitely long cylinder with the same relative complex index of refraction $m$. Figure $\underline{4}$ indicates that the scattering cross section of linear chains of spheres increased with an increasing number of spheres for all size parameters considered. In addition, as the number of spheres increased, the scattering cross section asymptotically converged toward that of randomly oriented and infinitely long volume-equivalent cylinders with diameter $d_{c, \mathrm{eq}, V}$.

Let us define the critical number of spheres $N_{s, \text { cr }}$ necessary to achieve an error less than $5 \%$ between the scattering cross sections of a linear chain of spheres and those of infinitely long cylinders. Figure $\underline{5}$ plots the critical number of spheres $N_{s, \mathrm{cr}}$ as a function of size parameter $\chi_{s}$. It is evident that $N_{s, \mathrm{cr}}$ decreased with increasing size parameter according to the power law

$$
N_{s, \mathrm{cr}}=K \chi_{s}^{p}
$$

where $K$ and $p$ are empirical constants found, by least square fitting, to be $K=136$ and $p=-0.7$ with a coefficient of determination $R^{2}=0.997$ estimated based on its most general
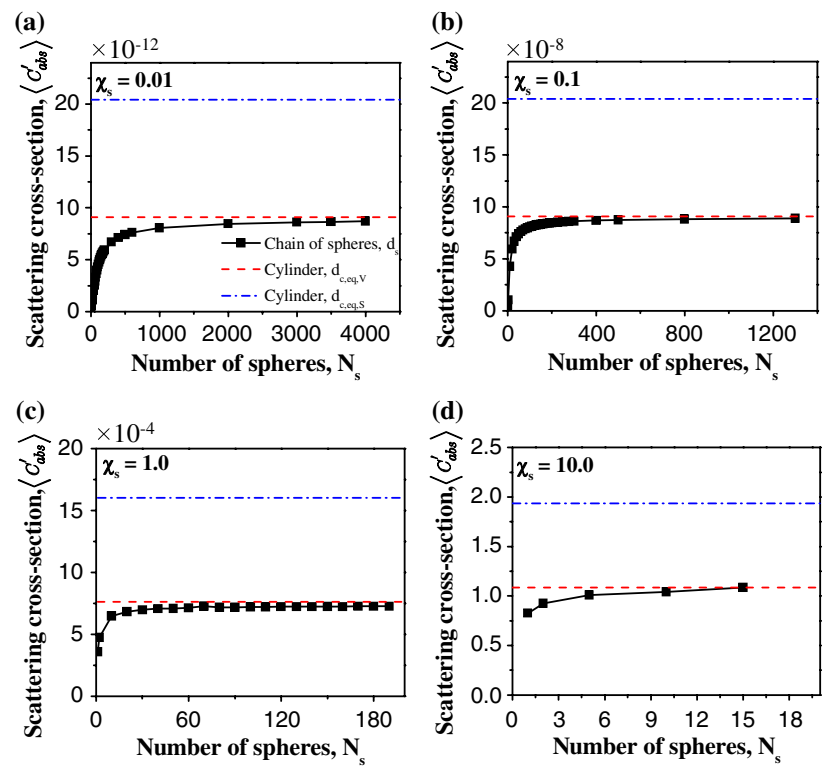

Fig. 4. Scattering cross section $\left\langle C_{\text {sca }}^{\prime}\right\rangle\left(m, \chi_{s}\right)$ per unit length (in $\mathrm{m}^{2} / \mathrm{m}$ ) for randomly oriented linear chains of monodisperse spheres as a function of the number of spheres $N_{s}$ and for randomly oriented and infinitely long equivalent cylinders of diameter $d_{c, \mathrm{eq}, S}$ and $d_{c, \text { eq }, V}$ for size parameter $\chi_{s}=0.01,0.1,1.0$, and 10.0 and $m=1.0165+i 0.003$.

definition $R^{2}=1-S S_{\text {res }} / S S_{\text {tot }}$, where $S S_{\text {res }}$ and $S S_{\text {tot }}$ are the residual sum of squares and the total sum of squares, respectively [49].

Finally, the cross sections and scattering matrix elements of linear chains of spheres were performed on a UCLA Hoffman 2 cluster with 20 parallel CPUs. It took about $5 \mathrm{~h}$ to simulate a linear chain of spheres with $N_{\mathrm{cr}}=190$ and $\chi_{s}=1$, while it took $30 \mathrm{~s}$ for the volume-equivalent cylinder. Similar speedup was observed for linear chains of spheres with other size parameters.

\section{B. Scattering Phase Function}

Figure $\underline{6}$ shows the scattering phase function $F_{11}(\Theta)$ as a function of scattering angle $\Theta$ for randomly oriented linear chains

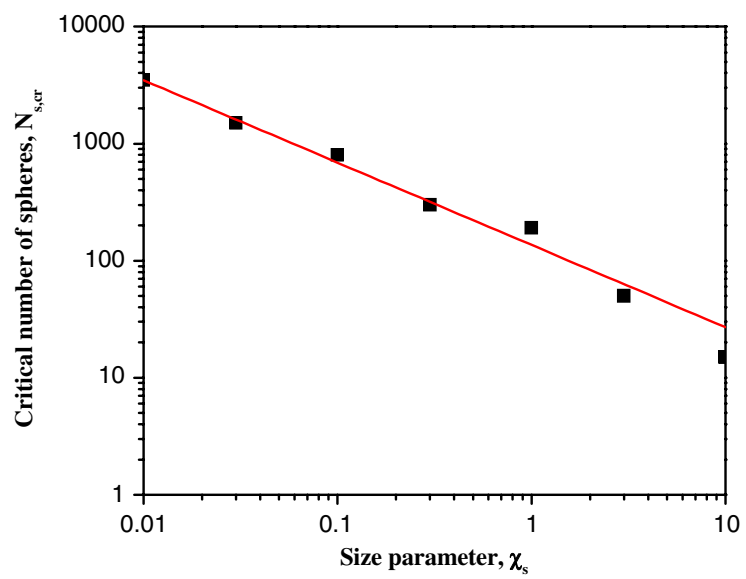

Fig. 5. Critical number of spheres $N_{s, \mathrm{cr}}$ beyond which the scattering cross section $\left\langle C_{\text {sca }}^{\prime}\right\rangle\left(m, \chi_{s}\right)$ of randomly oriented linear chains of spheres can be approximated as that of a randomly oriented and infinitely long volume-equivalent cylinder as a function of size parameter $\chi_{s}$. 

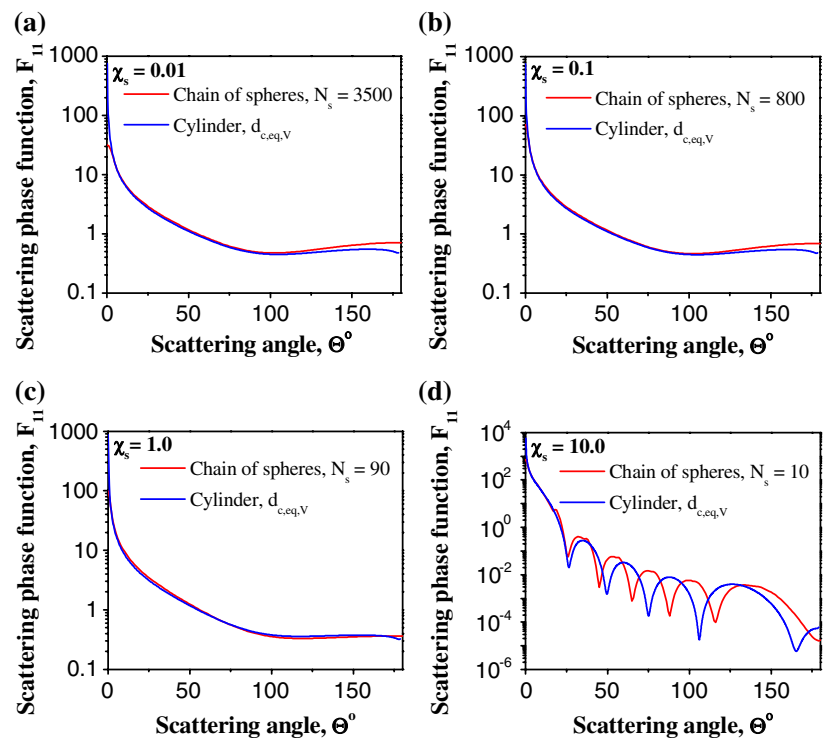

Fig. 6. Scattering phase functions $F_{11}(\Theta)$ of randomly oriented long linear chains consisting of $N_{s, c r}$ monodisperse spheres or diameter $d_{s}$ and randomly oriented and infinitely long volume-equivalent cylinders of diameter $d_{c \text { eq } V}$ as a function of scattering angle for $\chi_{s}=0.01,0.1$, 1.0 , and 10.0 and $m=1.0165+i 0.003$.

of spheres consisting of $N_{s, \mathrm{cr}}$ spheres with size parameter $\chi_{s}$ ranging between 0.01 and 10 . It also shows the phase function for the corresponding randomly oriented and infinitely long volume-equivalent cylinders. The value of $F_{11}\left(0^{\circ}\right)$ for linear chains of spheres increased from 31.54 to 721.8 as the size parameter $\chi_{s}$ increased from 0.01 to 10 . In other words, a linear chain of spheres scatters more and more strongly in the forward direction as the size parameter associated with individual constitutive spheres $\chi_{s}$ increases. Moreover, the scattering phase functions of the randomly oriented linear chain of spheres and infinitely long cylinders were very similar except in the forward and backward directions, $\Theta=0^{\circ}$ and $180^{\circ}$. In addition, the scattering phase function shows strong oscillations for scattering angles larger than $20^{\circ}$ for $\chi_{s}=10$. This indicates that resonance effects become increasingly important for large sphere size parameters. Table 1 compares $F_{11}\left(0^{\circ}\right)$ and the asymmetry factors $g_{s}$ and $g_{c, \mathrm{eq}, V}$ of a long $\left(N_{s}=N_{s, \mathrm{cr}}\right)$ randomly oriented linear chain of spheres with those of their volume-equivalent cylinder. It indicates that the values of $F_{11}\left(0^{\circ}\right)$ for linear chains of spheres and infinitely long cylinders were significantly different. However, $g_{s}$ and $g_{c, \text { eq,V }}$ differed by less than $1 \%$ and increased from 0.383 to 0.980 as $\chi_{s}$ increased from 0.01 to 10 .
Overall, the above results establish that the radiation characteristics for unpolarized radiation of long (i.e., $N_{s} \geq N_{s, \mathrm{cr}}$ ) randomly oriented linear chains of monodisperse spheres can be approximated as those of randomly oriented and infinitely long volume-equivalent cylinders. Then, their radiation characteristics can be computed using simple algorithm [1] instead of the superposition T-matrix method [22]. This simplifies and reduces significantly the computational effort.

For the practical problem of interest, filamentous cyanobacteria typically have size parameters larger than 10 and consist of more than 15 cells. Thus, the present study established that, as a first-order approximation, filamentous cyanobacteria in photobioreactors can be approximated as randomly oriented and infinitely long cylinders for the purpose of predicting their radiation characteristics for unpolarized incident radiation.

\section{Scattering Matrix Elements}

In cases concerned with polarized radiation, for the purpose of remote sensing, for example, detailed analysis of the normalized Stokes scattering matrix element is necessary. Figure 7 shows the ratios of the elements of the normalized Stokes scattering matrix (a) $-F_{12}(\Theta) / F_{11}(\Theta)$, (b) $F_{22}(\Theta) /$ $F_{11}(\Theta), \quad\left(\right.$ c) $\quad F_{33}(\Theta) / F_{11}(\Theta), \quad(d) \quad F_{44}(\Theta) / F_{11}(\Theta)$, and (e) $F_{34}(\Theta) / F_{11}(\Theta)$ as a function of scattering angle $\Theta$ for a long $\left(N_{s}=N_{s, \mathrm{cr}}\right)$ randomly oriented linear chain of spheres of diameter $d_{s}$ with size parameter $\chi_{s}$ equal to $0.01,0.1,1$, and 10. It also shows these ratios for the corresponding randomly oriented and infinitely long cylinders with volume-equivalent diameter $d_{c, \mathrm{eq}, V}=\sqrt{2 / 3} d_{s}$.

First, we observed that $\Delta\left(0^{\circ}\right)$ was equal to zero and that $F_{44}\left(180^{\circ}\right)=F_{11}\left(180^{\circ}\right)-2 F_{22}\left(180^{\circ}\right)$ for both long linear chains of spheres and infinitely long cylinders. We also verified that the results satisfied the symmetry relations for the scattering matrix elements of clusters of particles with one plane of symmetry expressed as [35] $F_{12}\left(0^{\circ}\right)=F_{12}\left(180^{\circ}\right)=F_{34}\left(0^{\circ}\right)=$ $F_{34}\left(180^{\circ}\right)=0, \quad F_{22}\left(0^{\circ}\right)=F_{33}(0), \quad F_{22}\left(180^{\circ}\right)=-F_{33}\left(180^{\circ}\right)$, $F_{11}\left(180^{\circ}\right)-F_{22}\left(180^{\circ}\right)=F_{44}\left(180^{\circ}\right)-F_{33}\left(180^{\circ}\right)$, and $F_{11}\left(180^{\circ}\right)-$ $F_{22}\left(180^{\circ}\right)=F_{44}\left(180^{\circ}\right)-F_{33}\left(180^{\circ}\right)$.

Second, it is worth noting that the ratios of the elements of the normalized Stokes scattering matrix for long linear chains of monodisperse spheres were nearly identical to one another for size parameter $\chi_{s}$ between 0.01 and 1 . The behavior of the matrix element ratios was very similar to the results reported (i) by Liu and Mishchenko [36] for orientation-averaged scattering matrix elements of fractal-like soot aggregates consisting of more than 200 monodisperse spherical monomers of diameter $d_{s}=20 \mathrm{~nm}$ with $m=1.75+0.435$ at $\lambda=628 \mathrm{~nm}$ $\left(\chi_{s}=0.1\right)$, and (ii) by Bunkin et al. [50] for ensemble-averaged

Table 1. Comparison between Selected Scattering Properties of Randomly Oriented Long Linear Chains of Spheres with Size Parameter $\chi_{s}$ Equal to 0.01, 0.1, 1.0, and 10.0 and their Randomly Oriented and Infinitely Long Volume-Equivalent Cylinders ${ }^{a}$

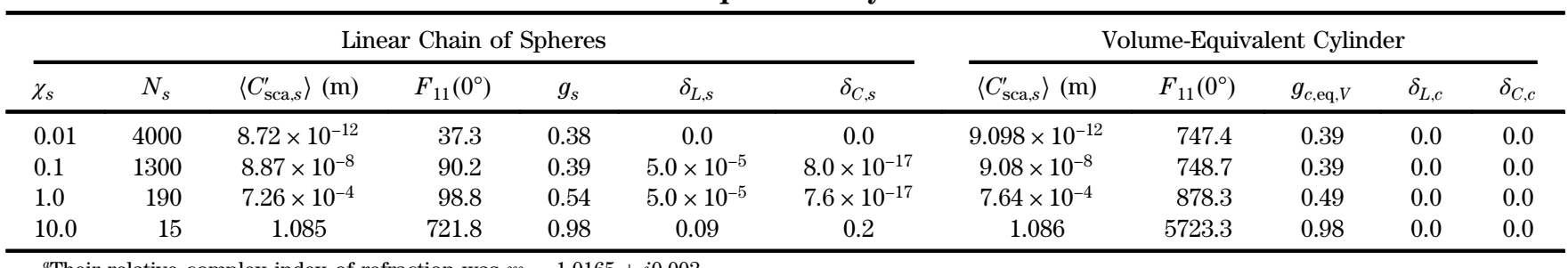

${ }^{a}$ Their relative complex index of refraction was $m=1.0165+i 0.003$. 

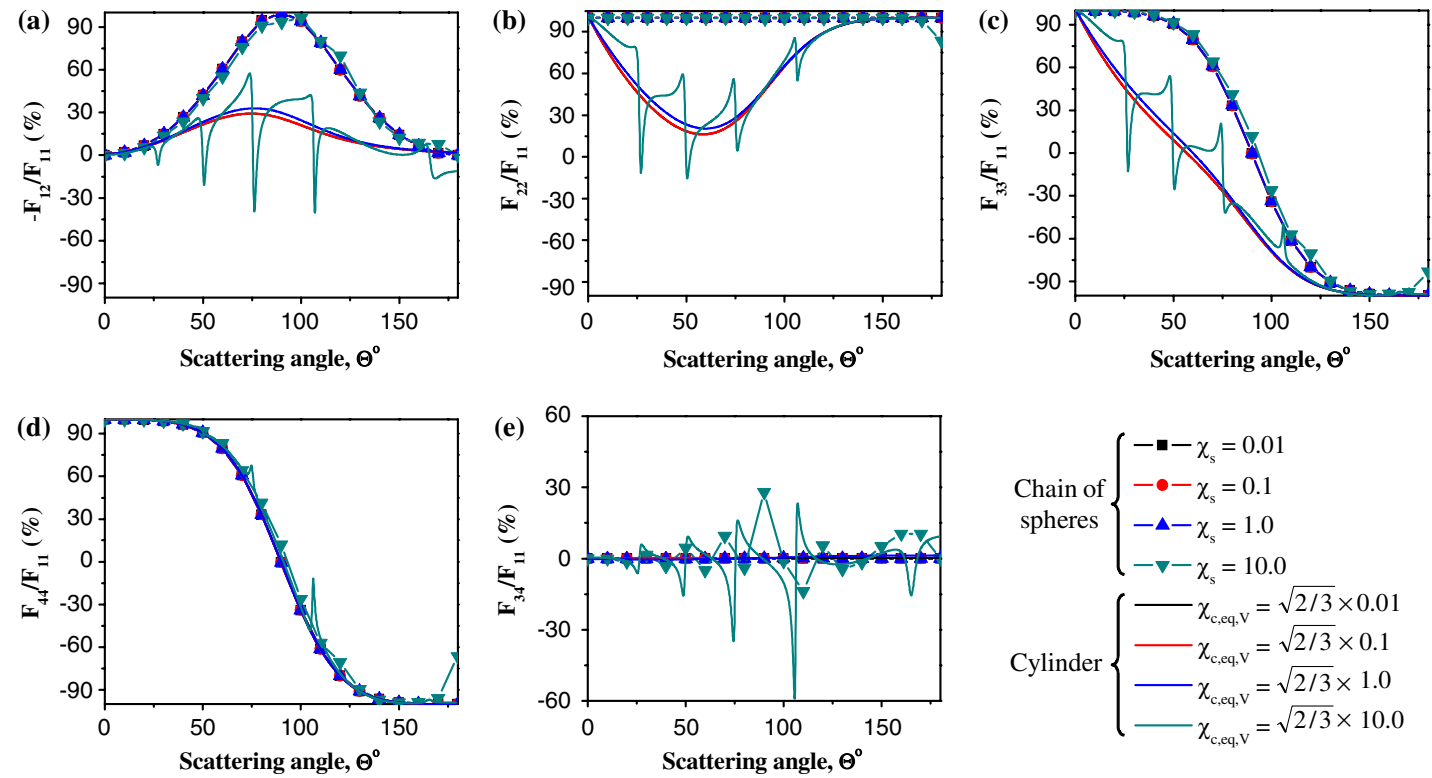

Fig. 7. Scattering matrix element $\operatorname{ratios}(\mathrm{a})-F_{12}(\Theta) / F_{11}(\Theta),(\mathrm{b}) F_{22}(\Theta) / F_{11}(\Theta),(\mathrm{c}) F_{33}(\Theta) / F_{11}(\Theta),(\mathrm{d}) F_{44}(\Theta) / F_{11}(\Theta)$, and $(\mathrm{e}) F_{34}(\Theta) / F_{11}(\Theta)$ as a function of scattering angle $\Theta$ for randomly oriented linear chains of spheres consisting of $N_{s, c r}$ monodisperse spheres of diameter $d_{s}$ and of infinitely long cylinders of volume-equivalent diameter as function of scattering angle for $\chi_{s}=0.01,0.1,1.0$, and 10 and $m=1.0165+i 0.003$.

scattering matrix elements of stochastic ensembles of nonabsorbing nanosphere clusters made of $500 \pm 70$ polydisperse monomers with mean diameter of $100 \mathrm{~nm}$ and $m=0.75$ at $\lambda=532 \mathrm{~nm}\left(\chi_{s} \sim 0.6\right)$.

Moreover, for a given value of $\chi_{s}$, increasing the numbers of spheres in the linear chain significantly affected the scattering phase function $F_{11}(\Theta)$ but not the ratios $F_{12}(\Theta) / F_{11}(\Theta)$, $F_{22}(\Theta) / F_{11}(\Theta), \quad F_{33}(\Theta) / F_{11}(\Theta), \quad F_{44}(\Theta) / F_{11}(\Theta), \quad$ and $F_{34}(\Theta) / F_{11}(\Theta)$. This was also observed for linear chains of spheres with large size parameter $\left(\chi_{s}=5\right)$ [20] and for fractal-like soot aggregates [36].

The degree of linear polarization of linear chains of spheres for unpolarized incident radiation $-F_{12}(\Theta) / F_{11}(\Theta)$ reached a maximum of $100 \%$ at scattering angle $\Theta$ around $90^{\circ}$. It also vanished in the forward scattering $\left(\Theta=0^{\circ}\right)$ and backscattering angles $\left(\Theta=180^{\circ}\right)$, as expected for a cluster of spheres with a plane of symmetry [20,51]. Similar results were obtained for a fractal cluster of soot particles and were attributed to the fact that scattering was dominated by individual Rayleigh-sized spheres [24,36]. In addition, the light scattered by multiple spheres was more linearly polarized than that by the volume-equivalent cylinder. In fact, $-F_{12}(\Theta) / F_{11}(\Theta)$ reached a maximum of about $30 \%-35 \%$ around $80^{\circ}$ for randomly oriented and infinitely long volume-equivalent cylinders and was nearly independent of $\chi_{s}$ except for $\chi_{s}=10$. In this latter case, $-F_{12}(\Theta) / F_{11}(\Theta)$ featured resonances at the same scattering angles as those observed in $F_{11}(\Theta)$.

The ratio $F_{22}(\Theta) / F_{11}(\Theta)$ was equal to $100 \%$ at all scattering angles for randomly oriented linear chains of spheres for any size parameters. These results were identical to those obtained with a single sphere and further confirm the above observations that the single spheres dominated scattering. However, the linear and circular polarization ratios $\delta_{L, s}$ and $\delta_{C, s}$ were nearly 0.0 for $\chi_{s} \leq 1$ and increased for larger size parameters $\chi_{s}$. Unlike single spheres, $\delta_{L, s}$ and $\delta_{C, s}$ were different for all size parameters, as summarized in Table 1. For infinitely long cylinders, $F_{22}(\Theta) / F_{11}(\Theta)$ was $100 \%$ for forward
$\left(\Theta=0^{\circ}\right)$ and backward $\left(\Theta=180^{\circ}\right)$ scattering angles but decreased between these two angles reaching a minimum of about $20 \%$ for a scattering angle around $50^{\circ}$. As previously mentioned, $\delta_{C, c}=\delta_{C, c}=0$ for randomly oriented and infinitely long cylinders of any size parameter.

For both randomly oriented cylinders and linear chains of spheres, the ratios $F_{33}(\Theta) / F_{11}(\Theta)$ and $F_{44}(\Theta) / F_{11}(\Theta)$ decreased from $100 \%$ to $-100 \%$ as the scattering angle increased from $0^{\circ}$ to $180^{\circ}$. The ratio $F_{33}(\Theta) / F_{11}(\Theta)$ decreased faster for a cylinder than for a linear chain of spheres. However, the ratio $F_{44}(\Theta) / F_{11}(\Theta)$ was nearly identical for randomly oriented linear chains of aligned spheres and infinitely long cylinders with volume-equivalent diameter for all size parameters $\chi_{s}$ considered. The ratios $F_{33}(\Theta) / F_{11}(\Theta)$ and $F_{44}(\Theta) / F_{11}(\Theta)$ for long linear chains of spheres were equal to each other and were identical to those of a single sphere. This was unlike what was observed in the validation cases for bispheres [19] and for linear chains of 1-5 spheres [20] with size parameters of 10 and 5 , respectively. Indeed, in these cases, the ratio $F_{22}(\Theta) / F_{11}(\Theta)$ departed from unity and the ratio $F_{33}(\Theta) / F_{11}(\Theta)$ was different from $F_{44}(\Theta) / F_{11}(\Theta)$, unlike those corresponding to a single sphere.

Finally, the normalized Stokes scattering matrix element ratio $F_{34}(\Theta) / F_{11}(\Theta)$ was equal to 0.0 for all scattering angles for $\chi_{s}=0.01,0.1$, and 1 for both randomly oriented linear chains of spheres and infinitely long cylinders. However, for $\chi_{s}=10$, $F_{34}(\Theta) / F_{11}(\Theta)$ featured several peaks with values between $-60 \%$ and $30 \%$ at scattering angles corresponding to the resonances observed in $F_{11}(\Theta)$ as well as in the other scattering element ratios. These resonance angles and the associated value of $F_{34}(\Theta) / F_{11}(\Theta)$ were significantly different from those for the volume-equivalent infinitely long cylinder.

Overall, the volume-equivalent cylinder featured normalized Stokes scattering element matrix ratios very different from those of linear chains of spheres. The equivalence observed for the absorption and scattering cross sections per unit length and for the asymmetric factor does not apply 
Table 2. Comparison of the Absorption and Scattering Cross Sections $\left\langle C_{\mathrm{abs}, s}\right\rangle$ and $\left\langle C_{\mathrm{sca}, s}\right\rangle$ of Two Randomly Oriented Linear Chains of Spheres (a) with Representative Arbitrary Diameter Distribution and (b) with Monodisperse Spheres with the Corresponding Average Diameter $d_{s}{ }^{a}$

\begin{tabular}{|c|c|c|c|c|c|c|c|}
\hline Chain No. & \multicolumn{4}{|c|}{ (a) Polydisperse Spheres } & \multicolumn{3}{|c|}{ (b) Monodisperse Spheres } \\
\hline 1 & $2.62-4.65$ & 0.48 & 0.136 & 0.179 & 3.32 & 0.122 & 0.170 \\
\hline
\end{tabular}

${ }^{a}$ The complex index of refraction was $m=1.0165+i 0.003$ for all spheres.

to the normalized Stokes scattering matrix element ratios other than $F_{44}(\Theta) / F_{11}(\Theta)$. These qualitative conclusions are expected to be valid for other linear chains of optically soft spheres.

\section{Effects of Polydispersity}

The radiation characteristics of linear chains of spheres can be affected by the spheres' polydispersity. To assess this effect in the context of filamentous cyanobacteria shown in Fig. 1, we considered two different linear chains of 14 spheres with average diameter $\bar{d}_{s}$ of 3.323 and $3.753 \mu \mathrm{m}$ and standard deviation $\sigma$ equal to 0.952 and $0.468 \mu \mathrm{m}$, respectively. The smallest and largest spheres were 2.617 and $4.655 \mu \mathrm{m}$ in diameter for a size parameter ranging between 6 and 10 . The size distributions of the two chains were representative of those measured for cyanobacterium Anaebena cylindrica. Here also, the relative complex index of refraction was $m=1.0165+i 0.003$ for all spheres.

Table 2 compares the absorption and scattering cross sections of the above-described randomly oriented linear chains of polydisperse spheres and those of the linear chain of monodisperse spheres with the corresponding average diameter $\bar{d}_{s}$. The results indicate that the cross sections $\left\langle C_{\mathrm{abs}, s}^{\prime}\right\rangle$ and $\left\langle C_{\mathrm{sca}, s}^{\prime}\right\rangle$ for chain 1 fell within $10 \%$ of those of a chain with monodisperse spheres of average diameter $\bar{d}_{s}$. The differences in $\left\langle C_{\mathrm{abs}, s}^{\prime}\right\rangle$ and $\left\langle C_{\mathrm{sca}, s}^{\prime}\right\rangle$ fell within $2 \%$ for chain 2 . This can be attributed to the narrower size distribution of chain 2 compared with chain 1 .

Overall, these results suggest that when estimating the absorption and scattering cross sections of filamentous cyanobacteria, the polydisperse vegetative cells and heterocysts can be treated as monodisperse with diameter equal to the average cell diameter $\bar{d}_{s}$.

\section{CONCLUSION}

This study presented predictions of the radiation characteristics and normalized Stokes scattering matrix elements of linear chains of spheres. The results established that scattering and absorption cross sections per unit length of randomly oriented linear chains of optically soft spheres and their asymmetry factors can be approximated as those of randomly oriented and infinitely long cylinders with volume-equivalent diameter provided that the number of spheres is larger than the critical sphere number $N_{s, \mathrm{cr}}=136 \chi_{s}^{-0.7}$. Finally, approximating long linear chains of spheres with infinitely long cylinders does not extend to the normalized Stokes scattering matrix element ratios. These results can be used for (i) predicting the radiation characteristics of filamentous cyanobacteria and (ii) retrieving the optical properties of filamentous cyanobacteria from experimental measurements of absorption and scattering cross sections.

\section{ACKNOWLEDGMENTS}

The computation for this study was performed on the Hoffman2 cluster hosted by the Academic Technology Services (ATS) at the University of California, Los Angeles. The authors also thank Prof. Pinar Mengüç for valuable discussions.

\section{REFERENCES}

1. M. Kerker, The Scattering of Light, and Other Electromagnetic Radiation (Academic, 1969).

2. C. F. Bohren and D. R. Huffman, Absorption and Scattering of Light by Small Particles (Wiley, 1998).

3. K. N. Liou, An Introduction to Atmospheric Radiation, 2nd ed. (Academic, 2002).

4. M. Jonasz and G. R. Fournier, Light Scattering by Particles in Water: Theoretical and Experimental Foundations (Academic, 2007).

5. M. I. Mishchenko and L. D. Travis, "Light scattering by polydispersions of randomly oriented spheroids with sizes comparable to wavelengths to observation," Appl. Opt. 33, 7206-7225 (1994).

6. M. I. Mishchenko and J. W. Hovenier, "Depolarization of light backscattered by randomly oriented nonspherical particles," Opt. Lett. 20, 1356-1358 (1995).

7. M. I. Mishchenko, L. D. Travis, and D. W. Mackowski, "T-matrix computations of light scattering by nonspherical particles: a review,” J. Quant. Spectrosc. Radiat. Transfer 55, 535-575 (1996).

8. M. I. Mishchenko, J. W. Hovenier, and L. D. Travis, Light Scattering by Nonspherical Particles (Academic, 2000).

9. D. W. Mackowski, "Discrete dipole moment method for calculation of the T matrix for nonspherical particles," J. Opt. Soc. Am. A 19, 881-893 (2002).

10. L. Bi, P. Yang, G. W. Kattawar, and M. I. Mishchenko, "A numerical combination of extended boundary condition method and invariant imbedding method applied to light scattering by large spheroids and cylinders," J. Quant. Spectrosc. Radiat. Transfer 123, 17-22 (2013).

11. E. M. Purcell and C. R. Pennypacker, "Scattering and absorption of light by nonspherical dielectric grains," Astrophys. J. 186, 705-714 (1973).

12. B. T. Draine, "The discrete-dipole approximation and its application to interstellar graphite grains,” Astrophys. J. 333, 848-872 (1988).

13. B. T. Draine and P. J. Flatau, "Discrete-dipole approximation for scattering calculations," J. Opt. Soc. Am. A 11, 1491-1499 (1994).

14. P. Yang and K. N. Liou, "Finite-difference time domain method for light scattering by small ice crystals in three-dimensional space," J. Opt. Soc. Am. A 13, 2072-2085 (1996).

15. P. Yang, G. W. Kattawar, and W. J. Wiscombe, "Effect of particle asphericity on single-scattering parameters: comparison between platonic solids and spheres," Appl. Opt. 43, 4427-4435 (2004).

16. M. I. Mishchenko and D. W. Mackowski, "Light scattering by randomly oriented bispheres," Opt. Lett. 19, 1604-1606 (1994).

17. D. W. Mackowski, "Calculation of total cross sections of multiplesphere clusters,” J. Opt. Soc. Am. A 11, 2851-2861 (1994).

18. M. I. Mishchenko, D. W. Mackowski, and L. D. Travis, "Scattering of light by bispheres with touching and separated components," Appl. Opt. 34, 4589-4599 (1995).

19. M. I. Mishchenko and D. W. Mackowski, "Electromagnetic scattering by randomly oriented bispheres: comparison of theory 
and experiment and benchmark calculations,” J. Quant. Spectrosc. Radiat. Transfer 55, 683-694 (1996).

20. D. W. Mackowski and M. I. Mishchenko, "Calculation of the T matrix and the scattering matrix for ensembles of spheres," J. Opt. Soc. Am. A 13, 2266-2278 (1996).

21. D. W. Mackowski, "A simplified model to predict the effects of aggregation on the absorption properties of soot particles," J. Quant. Spectrosc. Radiat. Transfer 100, 237-249 (2006).

22. D. W. Mackowski and M. I. Mishchenko, "A multiple sphere T-matrix Fortran code for use on parallel computer clusters," J. Quant. Spectrosc. Radiat. Transfer 112, 2182-2192 (2011).

23. L. Bi, P. Yang, G. W. Kattawar, and M. I. Mishchenko, "Efficient implementation of the invariant imbedding T-matrix method and the separation of variables method applied to large nonspherical inhomogeneous particles," J. Quant. Spectrosc. Radiat. Transfer 116, 169-183 (2013).

24. R. A. West, "Optical properties of aggregate particles whose outer diameter is comparable to the wavelength," Appl. Opt. 30, 5316-5324 (1991).

25. M. F. Iskander, H. Y. Chen, and J. E. Penner, "Optical scattering and absorption by branched chains of aerosols," Appl. Opt. 28, 3083-3091 (1989).

26. M. F. Iskander, H. Y. Chen, and J. E. Penner, "Resonance optical absorption by fractal agglomerates of smoke aerosols," Atmos. Environ. A 25, 2563-2569 (1991).

27. S. Manickavasagam and M. P. Mengüç, "Scattering matrix elements of fractal-like soot agglomerates," Appl. Opt. 36, 1337-1351 (1997).

28. F. M. Kahnert, J. J. Stamnes, and K. Stamnes, "Can simple particle shapes be used to model scalar optical properties of an ensemble of wavelength-sized particles with complex shapes?" J. Opt. Soc. Am. A 19, 521-531 (2002).

29. L. Pilon, H. Berberoğlu, and R. Kandilian, "Radiation transfer in photobiological carbon dioxide fixation and fuel production by microalgae," J. Quant. Spectrosc. Radiat. Transfer 112, 2639-2660 (2011)

30. M. T. Madigan and J. M. Martinko, Biology of Microorganisms (Pearson Prentice Hall, 2006)

31. L. J. Stal, "Cyanobacteria," in Algae and Cyanobacteria in Extreme Environments, J. Seckbach, ed., Vol. 11 of Cellular Origin, Life in Extreme Habitats and Astrobiology (Springer, 2007), pp. 659-680.

32. J. R. Benemann, "Hydrogen production by microalgae," J. Appl. Phycol. 12, 291-300 (2000).

33. M. I. Mishchenko, L. D. Travis, and A. A. Lacis, Scattering, Absorption, and Emission of Light by Small Particles (Cambridge University, 2001).

34. S. Manickavasagam and M. P. Mengüc, "Scattering-matrix elements of coated infinite-length cylinders," Appl. Opt. 37, 2473-2482 (1998).
35. J. W. Hovenier and D. W. Mackowski, "Symmetry relations for forward and backward scattering by randomly oriented particles,” J. Quant. Spectrosc. Radiat. Transfer 60, 483-492 (1998).

36. L. Liu and M. I. Mishchenko, "Effects of aggregation on scattering and radiative properties of soot aerosols," J. Geophys. Res. Atmos. 110, D11211 (2005).

37. S. C. Lee, "Radiative transfer through a fibrous medium: allowance for fiber orientation,” J. Quant. Spectrosc. Radiat. Transfer 36, 253-263 (1986).

38. A. C. Lind and J. M. Greenberg, "Electromagnetic scattering by obliquely oriented cylinders," J. Appl. Phys. 37, 3195-3203 (1966).

39. T. W. Tong and C. L. Tien, "Analytical models for thermal radiation in fibrous insulations," J. Build. Phys. 4, 27-44 (1980).

40. S. C. Lee, "Radiation heat-transfer model for fibers oriented parallel to diffuse boundaries," J. Thermophys. Heat Transfer 2, 303-308 (1988).

41. H. A. Yousif and E. Boutros, "A FORTRAN code for the scattering of EM plane waves by an infinitely long cylinder at oblique incidence," Comput. Phys. Commun. 69, 406-414 (1992).

42. M. F. Modest, Radiative Heat Transfer (Academic, 2003).

43. Z.-J. Li, Z.-S. Wu, Y. Shi, L. Bai, and H.-Y. Li, "Multiple scattering of electromagnetic waves by an aggregate of uniaxial anisotropic spheres," J. Opt. Soc. Am. A 29, 22-31 (2012).

44. D. Stramski, A. Bricaud, and A. Morel, "Modeling the inherent optical properties of the ocean based on the detailed composition of planktonic community," Appl. Opt. 40, 2929-2945 (2001).

45. J.-F. Cornet, C. G. Dussap, J. B. Gross, C. Binois, and C. Lasseur, "A simplified monodimensional approach for modeling coupling between radiant light transfer and growth kinetics in photobioreactors," Chem. Eng. Sci. 50, 1489-1500 (1995).

46. J. F. Cornet and C. G. Dussap, "A simple and reliable formula for assessment of maximum volumetric productivities in photobioreactors," Biotechnol. Progress 25, 424-435 (2009).

47. E. Lee, R.-L. Heng, and L. Pilon, "Spectral optical properties of selected photosynthetic microalgae producing biofuels," J. Quant. Spectrosc. Radiat. Transfer 114, 122-135 (2013).

48. S. C. Lee, "Scattering phase function for fibrous media," Int. J. Heat Mass Transfer 33, 2183-2190 (1990).

49. N. R. Draper and H. Smith, Applied Regression Analysis, 3rd ed. (Wiley, 1998).

50. N. F. Bunkin, A. V. Shkirin, N. V. Suyazov, and A. V. Starosvetskiy, "Calculations of light scattering matrices for stochastic ensembles of nanosphere clusters," J. Quant. Spectrosc. Radiat. Transfer 123, 23-29 (2013).

51. J. W. Hovenier, H. C. van de Hulst, and C. V. M. van der Mee, "Conditions for the elements of the scattering matrix," Astron. Astrophys. 157, 301-310 (1986). 